\title{
Amalgamated rings with clean-type properties
}

\author{
Negin Farshad $^{1}$ (D), Shaaban Ali SafariSabet*1 ${ }^{(1 D}$, Ahmad Moussavi ${ }^{2,3}$ \\ ${ }^{1}$ Department of Mathematics, Islamic Azad University, Central Tehran Branch, Tehran, Iran \\ ${ }^{2}$ Department of Mathematics, Islamic Azad University, South Tehran Branch, Tehran, Iran \\ ${ }^{3}$ Department of Pure Mathematics, Faculty of Mathematical Sciences, Tarbiat Modares University, \\ P.O.Box: 14115-134, Tehran, Iran
}

\begin{abstract}
Let $f: A \rightarrow B$ be a ring homomorphism and $K$ be an ideal of $B$. Many variations of the notions of clean and nil-clean rings have been studied by a variety of authors. We investigate strongly $\pi$-regular and clean-like properties of the amalgamation ring $A \bowtie^{f} K$ of $A$ with $B$ along $K$ with respect to $f$.
\end{abstract}

Mathematics Subject Classification (2020). 16N40, 16U40, 16 S99

Keywords. amalgamation ring, nil-clean ring, J-clean ring, semiclean ring, semiregular ring, exchange ring

\section{Introduction}

Throughout this paper all rings are associative with identity elements. Let $f: A \rightarrow B$ be a ring homomorphism and $K$ an ideal of $B$. In [14], M. D'Anna, C. A. Finocchiaro and M. Fontana, initiate a systematic study of a new subring of $A \times B$, defined by

$$
A \bowtie^{f} K:=\{(a, f(a)+k) \mid a \in A, k \in K\}
$$

called the amalgamation of $A$ with $B$ along $K$ with respect to $f$. This construction finds its roots in a paper by J. L. Dorroh appeared in 1932 and provides a general frame for studying the amalgamated duplication of a ring along an ideal, introduced and studied by D'Anna and Fontana in [11-13], and other classical constructions such as the $A+X B[X], A+$ $X B[[X]]$, the $C P I$-extensions of Boisen and Sheldon [4], the $D+M$ constructions and the Nagatas idealization [19].

Following [21], an element $a$ in a ring $R$ is called a clean element if $a$ is a sum of a unit and an idempotent in $R$. A ring is clean if all its elements are clean. Clean rings were initially developed by Nicholson [21] as a natural class of rings which have the exchange property.

In the past ten years, there have been many investigations concerning variants of the clean properties. Additionally, several authors have studied versions of such properties in the case of nil-clean rings. In [15] Diesl introduced nil-clean and strongly nil-clean rings,

\footnotetext{
*Corresponding Author.

Email addresses: neg.farshad.sci@iauctb.ac.ir; negin.farshad@gmail.com (N. Farshad),

sh_safarisabet@iauctb.ac.ir (Sh.A. Safarisabet), moussavi.a@modares.ac.ir; moussavi.a@azad.ac.ir (A. Moussavi)

Received: 19.01.2020; Accepted: 14.04.2021
} 
and he gave some basic examples and basic results on nil-clean and strongly nil-clean elements and rings. Following [15], an element $a \in R$ is called nil-clean if there is an idempotent $e \in R$ and a nilpotent element $b \in R$ such that $a=b+e$. The element $a$ is called strongly nil-clean if such an idempotent and nilpotent element can be chosen such that $b e=e b$. A ring $R$ is called nil-clean (respectively, strongly nil-clean) if every one of its elements is nil-clean (respectively, strongly nil-clean).

Following Nicholson [22], an element a in a ring $R$ is called right $\pi$-regular if it satisfies $a^{n} \in a^{n+1} R$ for some integer $n \geq 1$ or equivalently the condition that the chain $a R \supseteq$ $a^{2} R \supseteq \ldots$ terminates. The left $\pi$-regular elements are defined analogously. An element $a \in R$ is called strongly $\pi$-regular if it is both left and right $\pi$-regular; and $R$ is called a strongly $\pi$-regular ring if every element is strongly $\pi$-regular. By Diesl [15], it is shown that, an element $a \in R$ is strongly $\pi$-regular if and only if there is an idempotent $e \in R$ and a unit $u \in R$ such that $a=e+u, a e=e a$ and eae is nilpotent. An element $a \in R$ is called unipotent, if it can be written as $1+b$ for some nilpotent $b$. One of the results in [15] states that a ring $R$ is strongly nil-clean if and only if $R$ is strongly $\pi$-regular with $U(R)=1+\operatorname{nil}(R)$.

Călugăreanu in [5] introduced and studied $U U$ rings (rings whose units are unipotent). It is clear that nilpotent, idempotent and unipotent elements are strongly nil-clean. Thus, $U U$ rings can be viewed as the strong version of unit nil-clean rings. A ring $R$ is called a $U U$ ring if and only if every unit of $R$ is strongly nil-clean. This rings have been extensively investigated in Danchev and Lam [10], where, among others, they proved that a ring is strongly nil-clean if and only if it is an exchange (clean) $U U$ ring.

Chen in [6] defines an element $a \in R$ to be $J$-clean provided that there exist an idempotent $e \in R$ and an element $w$ in the Jacobson radical $J(R)$ such that $a=e+w$. The element $a$ is called strongly $J$-clean if such elements can be chosen such that $w e=e w$. A ring $R$ is $J$-clean (resp., strongly $J$-clean) in case every element in $R$ is $J$-clean (resp., strongly $J$-clean), see [6]. By [28] an element $r$ of a $\operatorname{ring} R$ is called semiclean if $r=p+u$, where $p$ is periodic, i.e., $p^{k}=p^{l}, p \in R$ for some positive integers $k$ and $l(k \neq l)$ and $u$ is a unit in $R$. A ring $R$ is called a semiclean ring if every element of $R$ is semiclean.

In [7], M. Chhiti, N. Mahdou and M. Tamekkante provided a characterization for clean property of $A \bowtie^{f} K$ and in [3], C. Bakkari and M. Es-Saidi gave a characterization for nil-clean property of $A \bowtie^{f} K$, where the rings are assumed to be commutative.

In this paper, we study many ring theoretical properties of the the amalgamation ring $A \bowtie^{f} K$, in the case where the rings are not assumed to be commutative. We give characterizations for the amalgamation ring $A \bowtie^{f} K$ to be nil-clean, strongly nil-clean, uniquely nil-clean, strongly $J$-clean, semiclean, strongly $\pi$-regular and unit unipotent, respectively. We then show that, when $A$ and $f(A)+K$ are $N R$ rings, then the amalgamation ring $A \bowtie^{f} K$ is $N R$ (i.e. $\operatorname{nil}(R)$ is a subring of $R$ ). We also provide necessary and sufficient conditions under which the amalgamated ring along an ideal $A \bowtie^{f} K$ is strongly $\pi$-regular. Finally the exchange property of the amalgamation ring $A \bowtie^{f} K$ is studied. This provides a new class of examples of rings with clean-like properties. Our results extend many existing results, e.g. Corollary 2.8 is a main result of [3].

We denote by $\operatorname{nil}(R), U(R), C(R), \operatorname{Idem}(R)$ and $J(R)$, the set of nilpotent elements, unit elements, central elements, the set of idempotents and the jacobson radical of $R$, respectively. 


\section{Clean like properties of amalgamated rings}

Let $A, B$ be rings which are not assumed to be commutative, $f: A \rightarrow B$ be a ring homomorphism and $K$ an ideal of $B$. We start with the following results for $\operatorname{Idem}\left(A \bowtie^{f}\right.$ $K), \operatorname{nil}\left(A \bowtie^{f} K\right), C\left(A \bowtie^{f} K\right)$ and $U\left(A \bowtie^{f} K\right)$, of the amalgamation $A \bowtie^{f} K$ of $A$ with $B$ along $K$ with respect to $f$. Recall that a ring $R$ is abelian if every idempotent of $R$ is central, that is $a e=e a$ for any $e \in \operatorname{Idem}(R), a \in R$.

Lemma 2.1. We have

$$
\operatorname{Idem}\left(A \bowtie^{f} K\right)=\{(e, f(e)+k) \mid e \in \operatorname{Idem}(A), f(e)+k \in \operatorname{Idem}(f(A)+K)\} .
$$

Lemma 2.2. Let $B$ be an abelian ring. Then

$$
\operatorname{Idem}(K)=0 \text { if and only if } \operatorname{Idem}\left(A \bowtie^{f} K\right)=\{(e, f(e)) \mid e \in \operatorname{Idem}(A)\} .
$$

Proof. Let $\operatorname{Idem}(K)=0$ and $(e, f(e)+k) \in \operatorname{Idem}\left(A \bowtie^{f} K\right)$. So $e^{2}=e$ and $(f(e)+k)^{2}=$ $f(e)+k$ and hence $e \in \operatorname{Idem}(A)$ and $f(e)+k=f(e)+2 f(e) k+k^{2}$, since $B$ is abelian. Thus $k-k^{2}=2 f(e) k$. So $f(e)\left(k-k^{2}\right)=2 f(e)^{2} k=2 f(e) k$ and hence $-f(e) k^{2}=f(e) k$. Therefore, $(f(e) k)^{4}=\left(f(e)^{2} k^{2}\right)^{2}=\left(f(e) k^{2}\right)^{2}=(-f(e) k)^{2}=(f(e) k)^{2}$, and that $f(e) k^{2}=$ $(f(e) k)^{2} \in \operatorname{Idem}(K)=\{0\}$. Hence $f(e) k=-f(e) k^{2}=0$, so $k-k^{2}=2 f(e) k=0$ and that $k=k^{2}$. Therefore $k \in \operatorname{Idem}(K)=\{0\}$. Consequently, $k=0$. Accordingly, $\operatorname{Idem}\left(A \bowtie^{f} K\right) \subseteq\{(e, f(e)) \mid e \in \operatorname{Idem}(A)\}$.

Conversely, let $\operatorname{Idem}(K) \neq 0$. So there exists $k \in K$ such that $k^{2}=k$. Then $(0, k)^{2}=$ $(0, k) \in \operatorname{Idem}\left(A \bowtie^{f} K\right)$, which is a contradiction.

In the following example we show that the abelian condition in the above lemma is a necessary condition.

Example 2.3. Let $R$ be a ring and $A$ be the triangular matrix $\operatorname{ring} T_{2}(R), B$ be the polynomial ring $T_{2}(R)[x]$ and $K$ the ideal $x T_{2}(R)[x]$ of $B$. Assume that $f: A \rightarrow B$ be defined by $f(M)=M$ for $M \in A$. As $\left(\left(\begin{array}{ll}1 & 0 \\ 0 & 0\end{array}\right)+\left(\begin{array}{ll}0 & 1 \\ 0 & 0\end{array}\right) x\right)^{2}=\left(\begin{array}{ll}1 & 0 \\ 0 & 0\end{array}\right)+\left(\begin{array}{ll}0 & 1 \\ 0 & 0\end{array}\right) x$ is an idempotent in $f(A)+K$, so $\operatorname{Idem}(f(A)+K) \neq \operatorname{Idem}(A)$. On the other hand $A \bowtie^{f} K \simeq f(A)+K$ so we have $\operatorname{Idem}\left(A \bowtie^{f} K\right) \neq\{(e, f(e)) \mid e \in \operatorname{Idem}(A)\}$.

According to Diesl [15], an element $a \in R$ is called uniquely nil-clean if there is a unique idempotent $e$ such that $a-e$ is nilpotent. A ring is called uniquely nil-clean if each of its elements is uniquely nil-clean.

Corollary 2.4. (1) If $B$ is abelian and $K \subseteq J(B)$, then

$$
\operatorname{Idem}\left(A \bowtie^{f} K\right)=\{(e, f(e)) \mid e \in \operatorname{Idem}(A)\} .
$$

(2) If $B$ is uniquely nil-clean and $\operatorname{Idem}(K)=0$, then

$$
\operatorname{Idem}\left(A \bowtie^{f} K\right)=\{(e, f(e)) \mid e \in \operatorname{Idem}(A)\} .
$$

Proof. (2) As every idempotent of uniquely nil-clean ring is central, [15, Lemma 5.5], so the proof follows from Lemma 2.2 .

Lemma 2.5. We have the following statements.

(1) $\operatorname{nil}\left(A \bowtie^{f} K\right)=\{(a, f(a)+k) \mid a \in \operatorname{nil}(A), f(a)+k \in \operatorname{nil}(f(A)+K)\}$.

(2) $U\left(A \bowtie^{f} K\right)=\{(a, f(a)+k) \mid a \in U(A), f(a)+k \in U(f(A)+K)\}$.

(3) $C\left(A \bowtie^{f} K\right)=\{(a, f(a)+k) \mid a \in C(A), f(a)+k \in C(f(A)+K)\}$.

Corollary 2.6. If $A$ and $f(A)+K$ are nil-clean rings, then $C\left(A \bowtie^{f} K\right)$ is a strongly nil-clean ring.

Proof. Let $(a, f(a)+k) \in C\left(A \bowtie^{f} K\right)$. By Lemma 2.5, $a \in C(A)$ and $f(a)+k \in$ $C(f(A)+K)$. Then $a$ and $f(a)+k$ are strongly nil-clean elements. Hence by [24, Lemma 2.4], $a-a^{2} \in \operatorname{nil}(A)$ and $(f(a)+k)-(f(a)+k)^{2} \in \operatorname{nil}(f(A)+K)$. Thus $(a, f(a)+$ $k)-(a, f(a)+k)^{2}=\left(a-a^{2}, f(a)+k-(f(a)+k)^{2}\right) \in \operatorname{nil}\left(A \bowtie^{f} K\right)$, by Lemma 2.5 . 
So by [24, Lemma 2.4], $(a, f(a)+k)$ is strongly nil-clean. Thus $C\left(A \bowtie^{f} K\right)$ is strongly nil-clean.

Proposition 2.7. For the amalgamation ring $A \bowtie^{f} K$, we have:

(1) If $A \bowtie^{f} K$ is a nil-clean (respectively, strongly nil-clean) ring, then $A$ and $f(A)+K$ are nil-clean (respectively, strongly nil-clean) rings.

(2) Assume that $\frac{f(A)+K}{K}$ is a uniquely nil-clean (resp., uniquely strongly nil-clean) ring. If $A$ and $f(A)+K$ are nil-clean (resp., strongly nil-clean) rings, then $A \bowtie^{f} K$ is nil-clean (resp., strongly nil-clean) ring.

Proof. (1) By [14, Proposition 5.1], we have the natural projections $p_{A}: A \bowtie^{f} K \rightarrow A$ defined by $p_{A}(a, f(a)+k)=a$ and $p_{B}: A \bowtie^{f} K \rightarrow B$ with $p_{B}(a, f(a)+k)=f(a)+k$. Hence, the following canonical isomorphisms hold

$$
\frac{A \bowtie^{f} K}{\{0\} \times K} \simeq A \text { and } \frac{A \bowtie^{f} K}{f^{-1}(K) \times\{0\}} \simeq f(A)+K .
$$

By [15, Proposition 3.13], the factor ring of nil-clean (resp., strongly nil-clean) ring is nilclean (resp., strongly nil-clean). So $A$ and $f(A)+K$ are nil-clean (resp., strongly nil-clean) rings.

(2) Let $(a, f(a)+k) \in A \bowtie^{f} K$. We have $a=b+e$, where $b \in \operatorname{nil}(A)$ and $e \in$ $\operatorname{Idem}(A)$ and $f(a)+k=f(x)+k_{1}+f(y)+k_{2}$ with $f(x)+k_{1} \in \operatorname{nil}(f(A)+K)$ and $f(y)+k_{2} \in \operatorname{Idem}(f(A)+K)$. It is clear $\overline{f(a)}=\overline{f(a)+k} \in \frac{f(A)+K}{K}, \overline{f(x)}=\overline{f(x)+k_{1}} \in$ $\frac{n i l(f(A)+K)+K}{K} \subseteq \operatorname{nil}\left(\frac{f(A)+K}{K}\right)$ and $\overline{f(y)}=\overline{f(y)+k_{2}} \in \operatorname{Idem}\left(\frac{f(A)+K}{K}\right)$. From $a=b+e$ we have $\overline{f(a)}=\overline{f(b)}+\overline{f(e)}$. So $\overline{f(x)}+\overline{f(y)}=\overline{f(a)}=\overline{f(b)}+\overline{f(e)}$. Since $\frac{f(A)+K}{K}$ is uniquely nil-clean, $\overline{f(x)}=\overline{f(b)}, \overline{f(y)}=\overline{f(e)}$. So there exist $k^{\prime}, k^{\prime \prime} \in K$ such that $f(x)=$ $f(b)+k^{\prime}, f(y)=f(e)+k^{\prime \prime}$ and then $(a, f(a)+k)=\left(b, f(b)+k^{\prime}+k_{1}\right)+\left(e, f(e)+k^{\prime \prime}+k_{2}\right)$ is the sum of a nilpotent and an idempotent element in $A \bowtie^{f} K$.

As a consequence of Corollary 2.6 and Proposition 2.7, we obtain the main result, Theorem 2.1, of [3].

Corollary 2.8. Let $f: A \rightarrow B$ be a ring homomorphism of commutative rings $A$ and $B$ and let $K$ be an ideal of $B$. Then the following conditions are equivalent:

(1) $A \bowtie^{f} K$ is nil-clean.

(2) $A$ and $f(A)+K$ are nil-clean.

Corollary 2.9. Let $A$ be a nil-clean ring and $f(A)+K$ be a nil-clean reduced ring. Then $A \bowtie^{f} K$ is a nil-clean ring.

Proof. Since $f(A)+K$ is nil-clean and reduced, $f(A)+K$ is uniquely nil-clean, by [15, Theorem 5.8]. By [15, Corollary 5.10], every factor ring of a uniquely nil-clean ring is uniquely nil-clean, so $\frac{f(A)+K}{K}$ is uniquely nil-clean and hence the proof is complete by Proposition 2.7(2).

Theorem 2.10. Let $K \subseteq \operatorname{nil}(B)$. Then we have the following statements.

(1) If $A$ is nil-clean, then $A \bowtie^{f} K$ is nil-clean.

(2) Assume that $f: A \rightarrow B$ is a ring monomorphism. If $f(A)+K$ is nil-clean then $A \bowtie^{f} K$ is nil-clean.

(3) Assume that $K$ is nilpotent. Then $A \bowtie^{f} K$ is strongly nil-clean if and only if $A$ is strongly nil-clean. 
Proof. (1) Let $(a, f(a)+k) \in A \bowtie^{f} K$. Since $\frac{A \bowtie^{f} K}{\{0\} \times K} \simeq A$ is nil-clean, for $\overline{(a, f(a)+k)} \in$ $\frac{A \bowtie^{f} K}{\{0\} \times K}$ we can assume that $\overline{(a, f(a)+k)}=\overline{\left(e, f(e)+k^{\prime}\right)}+\overline{\left(n, f(n)+k^{\prime \prime}\right)}$, for some idempotent $\overline{\left(e, f(e)+k^{\prime}\right)}$ and some nilpotent $\overline{\left(n, f(n)+k^{\prime \prime}\right)}$ in $\frac{A \bowtie^{f} K}{\{0\} \times K}$. By [18, Theorem 21.28], we lift $\overline{\left(e, f(e)+k^{\prime}\right)}$ to an idempotent $\left(e_{1}, f\left(e_{1}\right)+i\right)$ in $A \bowtie^{f} K$. So $((a, f(a)+k)+$ $\{0\} \times K)-\left(\left(e_{1}, f\left(e_{1}\right)+i\right)+\{0\} \times K\right)=\left(n, f(n)+k^{\prime \prime}\right)+\{0\} \times K$. Therefore $((a, f(a)+k)-$ $\left.\left(e_{1}, f\left(e_{1}\right)+i\right)\right)+\{0\} \times K=\left(n, f(n)+k^{\prime \prime}\right)+\{0\} \times K$. Hence $(a, f(a)+k)-\left(e_{1}, f\left(e_{1}\right)+i\right)$ is nilpotent modulo $\{0\} \times K$, since $\{0\} \times K$ is nil, this means $(a, f(a)+k)-\left(e_{1}, f\left(e_{1}\right)+i\right)$ is nilpotent. That is $(a, f(a)+k)$ is nil-clean.

(2) As $K \subseteq \operatorname{nil}(B)$ and $f$ is a monomorphism, $f^{-1}(K) \subseteq \operatorname{nil}(A)$. On the other hand $\frac{A \bowtie^{f} K}{f^{-1}(K) \times\{0\}} \simeq f(A)+K$. The remaining proof is similar to $(1)$.

(3) The forward direction is shown in Proposition 2.7. Now let $(a, f(a)+k) \in A \bowtie^{f} K$. By the fact that $\frac{A \bowtie^{f} K}{\{0\} \times K} \simeq A$, we get $\overline{(a, f(a)+k)} \in \frac{A \bowtie^{f} K}{\{0\} \times K}$ is a strongly nil-clean element, as $A$ is strongly nil-clean. Thus by [15, Theorem 3.21], $(a, f(a)+k)$ is strongly nil-clean in $A \bowtie^{f} K$, and the result follows.

Corollary 2.11. Let $K \subseteq \operatorname{nil}(B)$.

(1) $A \bowtie^{f} K$ is nil-clean if and only if $A$ is nil-clean.

(2) Let $f: A \rightarrow B$ be a ring monomorphism. Then $A \bowtie^{f} K$ is nil-clean if and only if $f(A)+K$ is nil-clean.

The next example shows that the condition $K \subseteq \operatorname{nil}(B)$ in Theorem 2.10 is not redundant.

Example 2.12. Let $A$ be a nil-clean ring, $f: A \rightarrow A[x]$, with $f(a)=a$ for $a \in A$, be a ring homomorphism and $K$ be the ideal $x A[x]$ of $A[x]$. It is clear that $A[x]$ is not nil-clean and $K$ is not nil. So $A \bowtie^{f} K \simeq f(A)+K$ is not nil-clean.

By [1], the $n$ - $\varphi$-trivial extension (simply $n$-trivial extension) of $R$ by $M$ is the set denoted by $R \ltimes_{\varphi} M_{1} \ltimes \ldots \ltimes M_{n}$ (simply $R \ltimes_{n} M_{1} \ltimes \ldots \ltimes M_{n}$ ), where $R$ is a ring, $M=\left(M_{i}\right)_{i=1}^{n}$ is a family of $R$-modules, and $\varphi=\left\{\varphi_{i, j}\right\}_{1 \leq i, j \leq n-1, i+j \leq n}$ is a family of bilinear maps such that each $\varphi_{i, j}$ is written multiplicatively $\varphi_{i, j}: M_{i} \times M_{j} \rightarrow M_{i+j}, \varphi_{i, j}\left(m_{i}, m_{j}\right)=m_{i} m_{j}$. The underlying additive group of $n$-trivial extension is $R \oplus M_{1} \oplus \ldots \oplus M_{n}$, and the elements of $n$-trivial extension ring are multiplied as $\left(r, m_{1}, \ldots, m_{n}\right)\left(r^{\prime}, m_{1}^{\prime}, \ldots, m_{n}^{\prime}\right)=\left(r r^{\prime}, m_{1}^{\prime \prime}, \ldots, m_{n}^{\prime \prime}\right)$ where $m_{i}^{\prime \prime}=\sum_{j+k=i} m_{j} m_{k}^{\prime}$. Note that $R \ltimes_{n} M_{1} \ltimes \ldots \ltimes M_{n}$ is associative precisely when $\left(m_{i} m_{j}\right) m_{k}=m_{i}\left(m_{j} m_{k}\right)$ for $m_{i} \in M_{i}, m_{j} \in M_{j}$ and $m_{k} \in M_{k}$ with $1 \leq i, j, k \leq n-2$ and $i+j+k \leq n$. In terms of the pre-product maps, this means that $\varphi_{i+j, k}\left(\varphi_{i, j}\left(m_{i}, m_{j}\right), m_{k}\right)=$ $\varphi_{i, j+k}\left(m_{i}, \varphi_{j, k}\left(m_{j}, m_{k}\right)\right)$. The $n$-trivial extension $R \ltimes_{n} M_{1} \ltimes \ldots \ltimes M_{n}$ is an extension of the classical trivial extension of rings to extensions associated to $n$ modules for any integer $n \geq 1$.

As observed in [1], any $n$-trivial extension $R \ltimes_{n} M_{1} \ltimes \ldots \ltimes M_{n}$ can be seen as the amalgamation of $R$ with $R \ltimes_{n} M_{1} \ltimes \ldots \ltimes M_{n}$ along $0 \ltimes_{n} M_{1} \ltimes \ldots \ltimes M_{n}$ with respect to the canonical injection.

Example 2.13. Let $R$ be a ring and $M=\left(M_{i}\right)_{i=1}^{n}$ be a family of $R$-modules. The $n$-trivial extension ring $R \ltimes_{n} M_{1} \ltimes \ldots \ltimes M_{n}$ is nil-clean if and only if $R$ is a nil-clean ring.

Remark 2.14. (1) If $f^{-1}(K)=0$, then $A \bowtie^{f} K$ is nil-clean (resp., strongly nil-clean) if and only if $f(A)+K$ is nil-clean (resp., strongly nil-clean), see $\left(^{*}\right)$. 
(2) If $K=0$, then $A \bowtie^{f} K$ is nil-clean (resp., strongly nil-clean) if and only if $A$ is nil-clean (resp., strongly nil-clean), see $\left(^{*}\right)$.

By a straight forward computation, we can determine the jacobson radical of the amalgamated ring $A \bowtie^{f} K$ with the following.

Lemma 2.15. We have

$$
J\left(A \bowtie^{f} K\right)=\{(a, f(a)+k) \mid a \in J(A), f(a)+k \in J(f(A)+K)\} .
$$

Recall that an element $a \in R$ is $J$-clean provided that there exist an idempotent $e \in R$ and an element $w \in J(R)$ such that $a=e+w$. By Chen [6], the element $a$ is called strongly J-clean if such elements can be chosen such that we $=e w$, and a ring $R$ is Jclean (resp., strongly J-clean) if every element of $R$ is J-clean (resp., strongly J-clean). A ring $R$ is called uniquely strongly J-clean provided that each element in $R$ has a unique representation as the sum of an element of $J(R)$ and an idempotent element in $R$ which commute each other. Now we consider the $J$-clean property of the amalgamation ring $A \bowtie^{f} K$.

Proposition 2.16. For the amalgamation ring $A \bowtie^{f} K$, we have

(1) If $A \bowtie^{f} K$ is a J-clean (resp., strongly J-clean) ring, then $A$ and $f(A)+K$ are $J$-clean (resp., strongly J-clean) rings.

(2) Let $\frac{f(A)+K}{K}$ be uniquely strongly J-clean. Then $A \bowtie^{f} K$ is strongly J-clean if and only if $A$ and $f(A)+K$ are strongly $J$-clean.

Proof. The proof is similar to that of Proposition 2.7.

Corollary 2.17. If $f(A)+K$ and $A$ are uniquely nil-clean rings, then $A \bowtie^{f} K$ is strongly J-clean.

Proof. To prove the result, we make two claims;

Claim (1); $f(A)+K$ and $A$ are strongly J-clean. Indeed, as $f(A)+K$ and $A$ are uniquely nil-clean rings, $f(A)+K$ and $A$ are uniquely clean rings, by [15, Theorem 5.9] and so by [6, Corollary 2.4] these are strongly J-clean with all idempotents central.

Claim (2); $\frac{f(A)+K}{K}$ is uniquely strongly J-clean. As $f(A)+K$ is uniquely clean and as every factor ring of uniquely clean ring is uniquely clean, [23, Theorem 22], $\frac{f(A)+K}{K}$ is uniquely clean, and hence by [23, Theorem 20] and [6, Corollary 2.4], it is uniquely strongly J-clean.

Now the assumptions of Proposition 2.16 are satisfied. So $A \bowtie^{f} K$ is a strongly J-clean ring.

Theorem 2.18. Let $K \subseteq \operatorname{nil}(B)$. Then we have the following statements.

(1) If $A$ is J-clean then $A \bowtie^{f} K$ is J-clean.

(2) Assume that $f: A \rightarrow B$ is a ring monomorphism. If $f(A)+K$ is $J$-clean then $A \bowtie^{f} K$ is J-clean.

Proof. The proof is similar to that of Theorem 2.10.

Example 2.19. Let $R$ be a ring and $M=\left(M_{i}\right)_{i=1}^{n}$ be a family of $R$-modules. The $n$-trivial extension ring $R \ltimes_{n} M_{1} \ltimes \ldots \ltimes M_{n}$ is $J$-clean if and only if $R$ is a $J$-clean ring.

Example 2.20. Let $A$ be a strongly $J$-clean ring, $j: A \rightarrow A[[x]]$ be the natural embedding and $K$ be the ideal $x A[[x]]$ of $A[[x]]$. As every power series ring over a strongly J-clean ring is strongly $J$-clean, [6], we have $A[[x]]$ is strongly $J$-clean and therefore $A \bowtie^{j} K \simeq$ $A+X A[[x]]$ is a strongly $J$-clean ring.

Ye in [28] defined semiclean rings. By [28, Proposition 2.1], every factor ring of a semiclean ring is semiclean. Recall that a ring $R$ is a uniquely semiclean ring provided 
that each element in $R$ has a unique representation as the sum of a periodic element and a unit element.

Lemma 2.21. If $A$ and $f(A)+K$ are periodic rings, then $A \bowtie^{f} K$ is a periodic ring.

Proof. Let $(a, f(a)+k) \in A \bowtie^{f} K$. As $A$ and $f(A)+K$ are periodic rings so by [28, Lemma 5.2], there exist positive integers $t_{1}$ and $t_{2}$ such that $a^{t_{1}}$ is an idempotent in $A$ and $(f(a)+k)^{t_{2}}$ is an idempotent in $f(A)+K$. Now we have $(a, f(a)+k)^{t_{1} t_{2}}=$ $\left(\left(a^{t_{1}}\right)^{t_{2}},\left((f(a)+k)^{t_{2}}\right)^{t_{1}}\right)=\left(a^{t_{1}},(f(a)+k)^{t_{2}}\right) \in \operatorname{Idem}\left(A \bowtie^{f} K\right)$, by Lemma 2.1. As every idempotent element is periodic, so $(a, f(a)+k)$ is a periodic element in $A \bowtie^{f} K$.

Proposition 2.22. We have the following statements.

(1) If $A \bowtie^{f} K$ is a semiclean ring, then $A$ and $f(A)+K$ are semiclean rings.

(2) Assume that $\frac{f(A)+K}{K}$ is uniquely semiclean. If $A$ and $f(A)+K$ are semiclean rings, then $A \bowtie^{f} K$ is semiclean.

Proof. (1) By [28, Proposition 2.1] every factor ring of a semiclean ring is semiclean, so the proof is the same as the proof (1) in Proposition 2.7.

(2) Let $(a, f(a)+k) \in A \bowtie^{f} K$. We have $a=p+u$ with $p$ a periodic element of $A$ and $u \in U(A)$, and $f(a)+k=f(q)+k_{1}+f(s)+k_{2}$ with $f(q)+k_{1}$ a periodic element of $f(A)+K$ and $f(s)+k_{2} \in U(f(A)+K)$, since $A$ and $f(A)+K$ are semiclean rings. It is clear that $\overline{f(a)}=\overline{f(a)+k} \in \frac{f(A)+K}{K}$ and $\overline{f(q)}=\overline{f(q)+k_{1}}$ is a periodic element of $\frac{f(A)+K}{K}$ and $\overline{f(s)}=\overline{f(s)+k_{2}} \in U\left(\frac{f(A)+K}{K}\right)$. From $a=p+u$ we have $\overline{f(a)}=\overline{f(p)}+\overline{f(u)}$, so $\overline{f(q)}+\overline{f(s)}=\overline{f(a)}=\overline{f(p)}+\overline{f(u)}$. Since $\frac{f(A)+K}{K}$ is a uniquely semiclean ring, $\overline{f(q)}=$ $\overline{f(p)}, \overline{f(s)}=\overline{f(u)}$. So there exist $k^{\prime}, k^{\prime \prime} \in K$ such that $f(q)=f(p)+k^{\prime}, f(s)=f(u)+k^{\prime \prime}$. Thus $(a, f(a)+k)=\left(p, f(p)+k^{\prime}+k_{1}\right)+\left(u, f(u)+k^{\prime \prime}+k_{2}\right)$ is the sum of a periodic element, by Lemma 2.21, and a unit element in $A \bowtie^{f} K$.

Let $I$ be an ideal of a ring $R$. Recall that a periodic element can be lifted modulo $I$, if for any $a \in R$ with $a^{k}-a^{l} \in I$, there exists $b \in R$ such that $b^{k}=b^{l} \in R$ and $a-b \in I$, see [28].

Now we give the following characterization of semiclean amalgamated rings.

Theorem 2.23. Let $K \subseteq \operatorname{nil}(B)$. Then we have the following statements.

(1) Assume that periodic elements in $A \bowtie^{f} K$ can be lifted modulo $\{0\} \times K$. If $A$ is semiclean then $A \bowtie^{f} K$ is semiclean.

(2) Assume that $f: A \rightarrow B$ is a ring monomorphism and periodic elements in $A \bowtie^{f} K$ can be lifted modulo $f^{-1}(K) \times\{0\}$. If $f(A)+K$ is semiclean then $A \bowtie^{f} K$ is semiclean.

Proof. (1) Let $(a, f(a)+k) \in A \bowtie^{f} K$. The proof follows from [28, Proposition 2.2], as $\frac{A \bowtie^{f} K}{\{0\} \times K} \simeq A$ is semiclean, $\{0\} \times K \subseteq J\left(A \bowtie^{f} K\right)$ and the periodic elements can be lifted modulo $\{0\} \times K$.

(2) Let $(a, f(a)+k) \in A \bowtie^{f} K$. The proof follows from [28, Proposition 2.2], as $\frac{A \bowtie^{f} K}{f^{-1}(K) \times\{0\}} \simeq f(A)+K$ is semiclean, $f^{-1}(K) \times\{0\} \subseteq J\left(A \bowtie^{f} K\right)$ and the periodic elements can be lifted modulo $f^{-1}(K) \times\{0\}$.

Corollary 2.24. Let $K \subseteq n i l(B)$ and assume that periodic elements in $A \bowtie^{f} K$ can be lifted modulo $\{0\} \times K$. Then $A \bowtie^{f} K$ is semiclean if and only if $A$ is semiclean.

Corollary 2.25. Let $K \subseteq \operatorname{nil}(B), f: A \rightarrow B$ be a ring monomorphism and assume that periodic elements in $A \bowtie^{f} K$ can be lifted modulo $f^{-1}(K) \times\{0\}$. Then $A \bowtie^{f} K$ is semiclean if and only if $f(A)+K$ is semiclean. 
Example 2.26. Let $A$ be a semiclean ring and $j: A \rightarrow A[[x]]$ be the natural embedding and $K:=x A[[x]]$. By [28, Proposition 3.3], every power series ring over a semiclean ring is semiclean. So $A[[x]]$ is semiclean and therefore $A \bowtie^{j} K \simeq A+X A[[x]]$ is a semiclean ring.

\section{Strongly $\pi$-regular amalgamated rings}

Following Nicholson [22], an element a in a ring $R$ is called right $\pi$-regular if it satisfies the following equivalent conditions:

(1) $a^{n} \in a^{n+1} R$ for some integer $n \geq 1$.

(2) $a^{n} R=a^{n+1} R$ for some integer $n \geq 1$.

(3) The chain $a R \supseteq a^{2} R \supseteq \ldots$ terminates.

The left $\pi$-regular elements are defined analogously.

Dischinger's Lemma. [16, Theorem 1] If every element of a ring $R$ is right $\pi$-regular then every element is left $\pi$-regular.

An element $a \in R$ is called strongly $\pi$-regular if it is both left and right $\pi$-regular; and $R$ is called a strongly $\pi$-regular ring if every element is strongly $\pi$-regular. By Diesl [15], it is shown that, an element $a \in R$ is strongly $\pi$-regular if and only if there is an idempotent $e \in R$ and a unit $u \in R$ such that $a=e+u, a e=e a$ and eae is nilpotent.

Theorem 3.1. The amalgamation ring $A \bowtie^{f} K$ is strongly $\pi$-regular if and only if $A$ and $f(A)+K$ are strongly $\pi$-regular rings.

Proof. Assume that $A$ and $f(A)+K$ are strongly $\pi$-regular rings and $(a, f(a)+k) \in A \bowtie^{f}$ $K$. We show that the chain of ideals $(a, f(a)+k) A \bowtie^{f} K \supseteq(a, f(a)+k)^{2} A \bowtie^{f} K \supseteq \ldots$ will terminate. We have two chains $a A \supseteq a^{2} A \supseteq \ldots$ and $(f(a)+k)(f(A)+K) \supseteq$ $(f(a)+k)^{2}(f(A)+K) \supseteq \ldots$ of $A$ and $f(A)+K$, respectively. So there exist positive integers $n$ and $m$ such that $a^{n} A=a^{n+1} A=a^{n+2} A=\ldots$ and $(f(a)+k)^{m}(f(A)+K)=$ $(f(a)+k)^{m+1}(f(A)+K)=\left((f(a)+k)^{m+2}(f(A)+K)=\ldots\right.$, since $A$ and $f(A)+K$ are strongly $\pi$-regular. Set $t=n m$, therefore for every $(b, f(b)+i) \in A \bowtie^{f} K$, we get $(a, f(a)+k)^{t}(b, f(b)+i)=\left(a^{t},(f(a)+k)^{t}\right)(b, f(b)+i)=\left(a^{t} b,(f(a)+k)^{t}(f(b)+\right.$ $i))=\left(a^{t+1} b,(f(a)+k)^{t+1}(f(b)+i)\right)=(a, f(a)+k)^{t+1}(b, f(b)+i)$. Then we have $(a, f(a)+k)^{t} A \bowtie^{f} K=(a, f(a)+k)^{t+1} A \bowtie^{f} K=\ldots$ and hence $A \bowtie^{f} K$ is a strongly $\pi$-regular ring.

Conversely, let $A \bowtie^{f} K$ be a strongly $\pi$-regular ring. We have $\frac{A \bowtie^{f} K}{\{0\} \times K} \simeq A$ and $\frac{A \bowtie^{f} K}{f^{-1}(K) \times\{0\}} \simeq f(A)+K$. Since by [26], every factor ring of a strongly $\pi$-regular ring is strongly $\pi$-regular, the proof is complete.

Next we study necessary and sufficient conditions under which the amalgamation ring along an ideal $A \bowtie^{f} K$ is a UU ring (i.e; every unit of $R$ is ring unipotent).

Theorem 3.2. We have the following statements.

(1) If $A$ and $f(A)+K$ are $U U$ rings, then $A \bowtie^{f} K$ is a $U U$ ring.

(2) If $A \bowtie^{f} K$ is a UU ring, then $A$ is a UU ring.

(3) Let $f: A \rightarrow B$ be a ring monomorphism and $K$ a nil ideal of $B$. If $A \bowtie^{f} K$ is a $U U$ ring, then $f(A)+K$ is a $U U$ ring.

Proof. (1) Let $(u, f(u)+k) \in U\left(A \bowtie^{f} K\right)$. So by Lemma 2.5, $u \in U(A)$ and $f(u)+k \in$ $U(f(A)+K)$. Since $A$ and $f(A)+K$ are UU rings, $u-1 \in \operatorname{nil}(A)$ and $f(u)+k-1 \in$ $\operatorname{nil}(f(A)+K)$. So $(u, f(u)+k)-(1,1) \in \operatorname{nil}\left(A \bowtie^{f} K\right)$. Thus $A \bowtie^{f} K$ is a UU ring.

(2) Let $u \in U(A)$. Then $(u, f(u)) \in U\left(A \bowtie^{f} K\right)$. Since $A \bowtie^{f} K$ is a UU ring, $(u, f(u))-$ 
$(1,1) \in \operatorname{nil}\left(A \bowtie^{f} K\right)$ and hence by Lemma $2.5, u-1 \in \operatorname{nil}(A)$. So the proof is complete. (3) Let $f(u)+k \in U(f(A)+K)$. Since $k \in K$ and $K \subseteq \operatorname{nil}(B)$ so $f(u)+k-k \in$ $U(f(A)+K)$. Hence $f(u) \in U(f(A)+K)$ and that $u \in U(A)$. Therefore by Lemma 2.5, $(u, f(u)+k) \in U\left(A \bowtie^{f} K\right)$. As $A \bowtie^{f} K$ is UU, $(u, f(u)+k)-(1,1) \in \operatorname{nil}\left(A \bowtie^{f} K\right)$. So $(u-1, f(u)+k-1) \in \operatorname{nil}\left(A \bowtie^{f} K\right)$. Thus by Lemma 2.5, $f(u)+k-1 \in \operatorname{nil}(f(A)+K)$.

By [15, Corollary 3.11], $R$ is a strongly nil-clean ring if and only if $R$ is a strongly $\pi$-regular and UU ring. So by Theorem 3.1 and Theorem 3.2, if $A$ and $f(A)+K$ are UU and strongly $\pi$-regular rings, then $A \bowtie^{f} K$ is a strongly nil-clean ring.

Remark 3.3. Consider the amalgamated ring $A \bowtie^{f} K$ of $A$ and $B$.

(1) If $B=K$ or $f: A \rightarrow B$ is an epimorphism, then $A \bowtie^{f} K$ is a UU ring if and only if $A$ and $B$ are UU rings, since in this case $A \bowtie^{f} K=A \times B$.

(2) If $f^{-1}(K)=0$, then $A \bowtie^{f} K$ is a UU ring if and only if $f(A)+K$ is a UU ring, see $(*)$.

(3) If $K=0$, then $A \bowtie^{f} K$ is a UU ring if and only if $A$ is a UU ring, see $\left(^{*}\right)$.

Recall by [8], that a ring $R$ forms an NR ring if $\operatorname{nil}(R)$ is a subring of $R$.

Lemma 3.4. If $B$ is an $N R$ ring, then

$$
\operatorname{nil}\left(A \bowtie^{f} K\right)=\{(a, f(a)+k) \mid a \in \operatorname{nil}(A), k \in \operatorname{nil}(K)\} .
$$

Proof. Let $a \in \operatorname{nil}(A)$ and $k \in \operatorname{nil}(K)$. Since $B$ is $N R, f(a)+k \in \operatorname{nil}(f(A)+K)$. So by Lemma 2.5, $(a, f(a)+k) \in \operatorname{nil}\left(A \bowtie^{f} K\right)$. Next consider $(a, f(a)+k) \in \operatorname{nil}\left(A \bowtie^{f} K\right)$. Therefore there exists a positive integer $n$ such that $(a, f(a)+k)^{n}=0$. Thus $a^{n}=0$ and $(f(a)+k)^{n}=0$. Hence $a \in \operatorname{nil}(A)$ and $f(a)+k \in \operatorname{nil}(B)$. Since $f(a) \in \operatorname{nil}(B)$ and $B$ is $N R, k \in \operatorname{nil}(B)$.

In [2], Antoin constructs various examples of Armendariz and related rings by reviewing and extending some results concerning the structure of $\operatorname{nil}(R)$. Anyway, the set of nilpotent elements forms a subring without unit, and this is true not only in the case of $R$ Armendariz, but in many of the generalizations of this condition.

Theorem 3.5. We have the following statements.

(1) If $A$ and $f(A)+K$ are NR rings, then $A \bowtie^{f} K$ is an NR ring.

(2) If $A \bowtie^{f} K$ is an NR ring, then so is $A$.

(3) Let $S$ be the set of regular central elements of $B$, such that $S \cap K \neq \varnothing$. If $A \bowtie^{f} K$ is $N R$, then $f(A)+K$ is $N R$.

Proof. (1) Let $(a, f(a)+k),\left(b, f(b)+k^{\prime}\right) \in \operatorname{nil}\left(A \bowtie^{f} K\right)$. So by Lemma 2.5, a,b $\operatorname{nil}(A)$ and $f(a)+k, f(b)+k^{\prime} \in \operatorname{nil}(f(A)+K)$. Since $A$ and $f(A)+K$ are NR, $a-b, a b \in \operatorname{nil}(A)$ and $(f(a)+k)-\left(f(b)+k^{\prime}\right),(f(a)+k)\left(f(b)+k^{\prime}\right) \in \operatorname{nil}(f(A)+K)$. So $(a, f(a)+k)-$ $\left(b, f(b)+k^{\prime}\right),(a, f(a)+k)\left(b, f(b)+k^{\prime}\right) \in \operatorname{nil}\left(A \bowtie^{f} K\right)$.

(2) Let $a, b \in \operatorname{nil}(A)$. We have $(a, f(a)),(b, f(b)) \in \operatorname{nil}\left(A \bowtie^{f} K\right)$. Since $A \bowtie^{f} K$ is NR, $(a, f(a))-(b, f(b)) \in \operatorname{nil}\left(A \bowtie^{f} K\right)$ and $(a, f(a))(b, f(b)) \in \operatorname{nil}\left(A \bowtie^{f} K\right)$. So by Lemma 2.5, $a-b \in \operatorname{nil}(A), a b \in \operatorname{nil}(A)$ and hence $A$ is NR.

(3) Let $f(a)+k, f(b)+k^{\prime} \in \operatorname{nil}(f(A)+K)$. We show that $(f(a)+k)-\left(f(b)+k^{\prime}\right) \in \operatorname{nil}(f(A)+$ $K)$ and $(f(a)+k)\left(f(b)+k^{\prime}\right) \in \operatorname{nil}(f(A)+K)$. We have $(0, e(f(a)+k)),\left(0, e\left(f(b)+k^{\prime}\right)\right) \in$ $\operatorname{nil}\left(A \bowtie^{f} K\right)$, for $e \in S \cap K$. Since $A \bowtie^{f} K$ is NR, $(0, e(f(a)+k))-\left(0, e\left(f(b)+k^{\prime}\right)\right) \in$ $\operatorname{nil}\left(A \bowtie^{f} K\right)$ and $(0, e(f(a)+k))\left(0, e\left(f(b)+k^{\prime}\right)\right) \in \operatorname{nil}\left(A \bowtie^{f} K\right)$. So by Lemma 2.5, $e\left((f(a)+k)-\left(f(b)+k^{\prime}\right)\right) \in \operatorname{nil}(f(A)+K), e^{2}\left((f(a)+k)\left(f(b)+k^{\prime}\right)\right) \in \operatorname{nil}(f(A)+K)$. Therefore there are positive integers $n$ and $m$, such that $\left(e\left((f(a)+k)-\left(f(b)+k^{\prime}\right)\right)\right)^{n}=0$ and $\left(e^{2}(f(a)+k)\left(f(b)+k^{\prime}\right)\right)^{m}=0$. Since $e$ is a regular central element, $((f(a)+k)-(f(b)+$ $\left.\left.k^{\prime}\right)\right)^{n}=0$ and $\left((f(a)+k)\left(f(b)+k^{\prime}\right)\right)^{m}=0$. Therefore $(f(a)+k)-\left(f(b)+k^{\prime}\right) \in \operatorname{nil}(f(A)+K)$ and $(f(a)+k)\left(f(b)+k^{\prime}\right) \in \operatorname{nil}(f(A)+K)$. 
Corollary 3.6. Let $S$ be the set of regular central elements of $B$, such that $S \cap K \neq \varnothing$. Then $A \bowtie^{f} K$ is $N R$ if and only if $f(A)+K$ and $A$ are $N R$.

Example 3.7. Let $R$ be an NR ring. Then by [8, Proposition 2.2] $A=\left\{\left(\begin{array}{ll}a & 0 \\ 0 & a\end{array}\right) \mid a \in R\right\}$, $B=\left\{\left(\begin{array}{cc}a & b \\ 0 & a\end{array}\right) \mid a, b \in R\right\}$ are NR rings. Let $f: A \rightarrow B$ be defined by $f\left(\begin{array}{ll}a & 0 \\ 0 & a\end{array}\right)=\left(\begin{array}{ll}a & 0 \\ 0 & a\end{array}\right)$ and $K=\left\{\left(\begin{array}{ll}0 & b \\ 0 & 0\end{array}\right) \mid b \in R\right\}$ be an ideal of $B$. Then $A \bowtie^{f} K \simeq f(A)+K$ is an NR ring.

Proposition 3.8. Let $A \bowtie^{f} K$ be an NR ring and $K$ be a nil ideal of $B$. Then $A$ is a $U U$ ring if and only if $A \bowtie^{f} K$ is a $U U$ ring.

Proof. Let $A$ be a UU ring. We have $\frac{A \bowtie^{f} K}{\{0\} \times K} \simeq A$. Since $K$ is a nil ideal of $B$ so $\{0\} \times K$ is a nil ideal of $A \bowtie^{f} K$. So by [5, Proposition 2.2], $A \bowtie^{f} K$ is a UU ring.

Proposition 3.9. Let $K$ be a nil ideal of $B, f: A \rightarrow B$ be a ring monomorphism and $A \bowtie^{f} K$ be an NR ring. If $f(A)+K$ is a UU ring, then $A \bowtie^{f} K$ is a UU ring.

Proof. Since $f$ is monomorphism and $K$ is a nil ideal, $f^{-1}(K)$ is a nil ideal of $A$ and hence $f^{-1}(K) \times 0$ is a nil ideal of $A \bowtie^{f} K$. On the other hand $\frac{A \bowtie^{f} K}{f^{-1}(K) \times\{0\}} \simeq f(A)+K$. So by [5, Proposition 2.2], $f(A)+K$ is a UU ring, and this implies that $A \bowtie^{f} K$ is a UU ring.

Let $R$ be a commutative ring with identity and $f=a_{0}+a_{1} x+\cdots+a_{n} x^{n} \in R[x]$. Then $f$ is a unit in $R[x]$ if and only if $a_{0}$ is a unit in $R$ and $a_{1}, a_{2}, \cdots, a_{n}$ are nilpotent elements in $R$; and $f$ is nilpotent in $R[x]$ if and only if all the coefficients are nilpotent. So by [5, Corollary 2.2], a polynomial ring $R[x]$ over a commutative ring with identity is UU if and only if $R$ is UU. So we have the following.

Example 3.10. Let $A$ be a commutative ring, $j: A \rightarrow A[x]$ the natural embedding and set $K:=x A[x]$. Then $A$ is a $\mathrm{UU}$ ring if and only if $A \bowtie^{j} K \simeq A+x A[x]$ is a UU ring.

\section{Exchange amalgamated rings}

An element $a$ in a ring $R$ is called von Neumann regular if $a=a b a$, for some $b \in R$, equivalently if the principal right ideal $a R$ is a direct summand of $R$, [18, Theorem 4.23]. A ring $R$ is called von Neumann regular if every element of $R$ is von Neumann regular.

Proposition 4.1. If $A \bowtie^{f} K$ is a von Neumann regular ring, then $A$ and $f(A)+K$ are von Neumann regular rings.

Proof. Let $a \in A$. We have $(a, f(a)) \in A \bowtie^{f} K$. Since $A \bowtie^{f} K$ is von Neumann regular, there exists $(b, f(b)+k) \in A \bowtie^{f} K$ such that $(a, f(a))=(a, f(a))(b, f(b)+$ $k)(a, f(a))=(a b a, f(a)(f(b)+k) f(a))$. So $a=a b a$ and hence $A$ is a von Neumann regular ring. Let $f(a)+k \in f(A)+K$. Then $(a, f(a)+k) \in A \bowtie^{f} K$. As $A \bowtie^{f} K$ is von Neumann regular, there exists $(b, f(b)+l) \in A \bowtie^{f} K$ such that $(a, f(a)+k)=$ $(a, f(a)+k)(b, f(b)+l)(a, f(a)+k)$. So $(a, f(a)+k)=(a b a,(f(a)+k)(f(b)+l)(f(a)+k))$ and then $f(a)+k=(f(a)+k)(f(b)+l)(f(a)+k)$. Hence $f(A)+K$ is von Neumann regular.

The following example shows that if $A$ is a von Neumann regular ring then the amalgamated ring $A \bowtie^{f} K$ is not necessarily von Neumann regular.

Example 4.2. Let $F$ be a field and $A=\left\{\left(\begin{array}{cc}a & 0 \\ 0 & a\end{array}\right) \mid a \in F\right\}, B=\left\{\left(\begin{array}{ll}a & b \\ 0 & a\end{array}\right) \mid a, b \in F\right\}$ and $K=\left\{\left(\begin{array}{ll}0 & b \\ 0 & 0\end{array}\right) \mid b \in F\right\}$ be an ideal of $B$. Consider $f: A \rightarrow B$ defined by $f\left(\begin{array}{ll}a & 0 \\ 0 & a\end{array}\right)=\left(\begin{array}{ll}a & 0 \\ 0 & a\end{array}\right)$. But $f(A)+K$ is not von Neumann regular, because for every $\left(\begin{array}{cc}r & s \\ 0 & r\end{array}\right) \in f(A)+K$ where $r, s \in F$ are non-zero, $\left(\begin{array}{ll}0 & b \\ 0 & 0\end{array}\right) \neq\left(\begin{array}{ll}0 & b \\ 0 & 0\end{array}\right)\left(\begin{array}{ll}r & s \\ 0 & r\end{array}\right)\left(\begin{array}{ll}0 & b \\ 0 & 0\end{array}\right)$. So $A \bowtie^{f} K \simeq f(A)+K$ is not von Neumann regular. 
Lemma 4.3. Let $f: A \rightarrow B$ be a ring homomorphism. If $K=0$ and $A$ is von Neumann regular then $A \bowtie^{f} K$ is von Neumann regular.

Proof. The proof is clear.

Proposition 4.4. [20, Proposition 2.2] The following are equivalent for an element a of a ring $R$.

(1) There exists $e^{2}=e \in a R$ such that $(1-e) a \in J(R)$.

(2) There exists $e^{2}=e \in a R$ such that $a(1-e) \in J(R)$.

(3) There exists a regular element $b \in R$ with $a-b \in J(R)$.

(4) There exists $b \in R$ with $b a b=b$ and $a-a b a \in J(R)$.

By Nicholson [20], an element $a$ of a ring $R$ is called semiregular, if it satisfies the above conditions. A ring is a semiregular ring if each of its elements is semiregular. Nicholson in [20, Theorem 2.9] shows that a ring $R$ is semiregular if $\frac{R}{J(R)}$ is von Neumann regular and idempotents lift modulo $J(R)$. The class of semiregular rings is very large. For example, every von Neumann regular ring is semiregular.

Proposition 4.5. If $A \bowtie^{f} K$ is semiregular, then $A$ and $f(A)+K$ are semiregular.

Proof. Let $a \in A$. Then $(a, f(a)) \in A \bowtie^{f} K$. As $A \bowtie^{f} K$ is semiregular, there exists $(e, f(e)+l)^{2}=(e, f(e)+l) \in(a, f(a)) A \bowtie^{f} K$ such that $((1,1)-(e, f(e)+l))(a, f(a)) \in$ $J\left(A \bowtie^{f} K\right)$. So $((1-e) a,(1-(f(e)+l)) f(a)) \in J\left(A \bowtie^{f} K\right)$. Hence by Lemma 2.15, $(1-e) a \in J(A)$ for some $e^{2}=e \in a A$.

Let $f(a)+k \in f(A)+K$. We have $(a, f(a)+k) \in A \bowtie^{f} K$. As $A \bowtie^{f} K$ is semiregular, there exists $(e, f(e)+l)^{2}=(e, f(e)+l) \in(a, f(a)+k) A \bowtie^{f} K$ such that $((1,1)-(e, f(e)+$ $l))(a, f(a)+k) \in J\left(A \bowtie^{f} K\right)$. By Lemma 2.15, $(1-(f(e)+l))(f(a)+k) \in J(f(A)+K)$ for some $(f(e)+l)^{2}=(f(e)+l) \in(f(a)+k)(f(A)+K)$.

Lemma 4.6. If $K$ is a nil ideal of $B$, then $J(f(A))+K \subseteq J(f(A)+K)$.

Proof. Let $f(a)+l \in J(f(A))+K$. We show that $f(a)+l \in J(f(A)+K)$. Assume by contrary that $f(a)+l \notin J(f(A)+K)$. So there exists $f(b)+t \in f(A)+K$ such that $1-(f(a)+l)(f(b)+t) \notin U(f(A)+K)$, by [18, Lemma 4.1]. As $f(a)+l \in J(f(A))+K$ and $f(a) \in J(f(A)), 1-f(a) f(b) \in U(f(A))$. So $1-f(a) f(b)-l f(b)=1-(f(a)+$ l) $f(b) \in U(f(A)+K)$. Thus $1-(f(a)+l)) f(b)-(f(a)+l)) t \in U(f(A)+K)$. Therefore $1-(f(a)+l)(f(b)+t) \in U(f(A)+K)$, a contradiction.

As by [20, Corollary 2.3], every homomorphic image of a semiregular ring is semiregular, so if $A$ is semiregular then $f(A)$ is semiregular.

Theorem 4.7. Let $K$ be a nil ideal of $B$ and $A$ be a semiregular ring. Then $A \bowtie^{f} K$ is a semiregular ring.

Proof. Let $(a, f(a)+k) \in A \bowtie^{f} K$. Since $f(a) \in f(A)$ is semiregular, there exists a von Neumann regular element $b \in A$ with $f(a)-f(b) \in J(f(A))$. So $f(a)-f(b)+k \in$ $J(f(A))+K \subseteq J(f(A)+K)$, by Lemma 4.6. Thus $(f(a)+k)-f(b) \in J(f(A)+K)$, for a von Neumann regular element $f(b) \in f(A)+K$. So for the von Neumann regular element $(b, f(b)) \in A \bowtie^{f} K,(a, f(a)+k)-(b, f(b)) \in J\left(A \bowtie^{f} K\right)$, by Lemma 2.15, and the result follows.

Corollary 4.8. Let $K$ be a nil ideal of $B$ and $A$ be a semiregular ring. Then $f(A)+K$ is semiregular.

Following Crawley and Jónsson [9], a module $M_{R}$ is said to have the exchange property if for every module $A_{R}$ and any two decompositions of $A_{R}, A_{R}=M_{R}^{\prime} \oplus N_{R}=\bigoplus_{i \in I} A_{i}$ with $M \cong M^{\prime}$, there exist submodules $A_{i}^{\prime} \subseteq A_{i}$ such that $A_{R}=M_{R}^{\prime} \oplus\left(\bigoplus_{i \in I} A_{i}^{\prime}\right)$. $M_{R}$ 
is said to have the finite exchange property if the above condition is satisfied whenever the index set $I$ is finite. A ring $R$ is said to be an exchange ring [27] if $R_{R}$ has the finite exchange property introduced by [9]. Warfield in [27] proved that this property is left-right symmetric. The class of exchange rings is quite large. It includes all semiperfect rings, all von Neumann regular rings, and all $\pi$-regular rings; see [27], [25].

Using [21, Proposition 1.1], we conclude the following.

Proposition 4.9. Let $A \bowtie^{f} K$ be an exchange ring. Then $A$ and $f(A)+K$ are exchange rings.

Proof. Let $a \in A$. Then $(a, f(a)) \in A \bowtie^{f} K$. As $A \bowtie^{f} K$ is exchange there exists $(e, f(e)+l)^{2}=(e, f(e)+l) \in A \bowtie^{f} K(a, f(a))$ such that $((1,1)-(e, f(e)+l)) \in A \bowtie^{f}$ $K\left((1,1)-(a, f(a))\right.$. So $\left((1-e),(1-(f(e)+l)) \in A \bowtie^{f} K((1,1)-(a, f(a))\right.$. Hence $(1-e) \in A(1-a)$ for some $e^{2}=e \in A a$.

Let $f(a)+k \in f(A)+K$. We have $(a, f(a)+k) \in A \bowtie^{f} K$. As $A \bowtie^{f} K$ is exchange there exists $(e, f(e)+l)^{2}=(e, f(e)+l) \in A \bowtie^{f} K(a, f(a)+k)$ such that $((1,1)-(e, f(e)+l)) \in$ $A \bowtie^{f} K((1,1)-(a, f(a)+k))$. So $(1-(f(e)+l)) \in(f(A)+K)(1-(f(a)+k))$, for some $(f(e)+l)^{2}=(f(e)+l) \in f(A)+K$.

Since by [21, Proposition 1.4], every homomorphic image of an exchange ring is exchange, so if $A$ is an exchange ring then $f(A)$ is an exchange ring.

Theorem 4.10. Let $K$ be a nil ideal of $B$ and $A$ be an exchange ring. Then $A \bowtie^{f} K$ is an exchange ring.

Proof. Let $(a, f(a)+k) \in A \bowtie^{f} K$. As $A$ is an exchange ring, $f(A)$ is exchange. So for $(f(e))^{2}=f(e) \in f(A) f(a)$ and $f(c) \in f(A)$, we have $(f(1)-f(e))-f(c)(f(1)-f(a)) \in$ $J(f(A))$. Then $(f(1)-f(e))-f(c)(f(1)-f(a))+f(c) k \in J(f(A))+K$ and hence by Lemma 4.6, $(1-f(e))-f(c)(1-(f(a)+k)) \in J(f(A)+K)$, for $(f(e))^{2}=f(e) \in(f(A)+K)(f(a)+k)$ and $f(c) \in f(A)+K$. Thus for $(e, f(e))^{2}=(e, f(e)) \in A \bowtie^{f} K$ and $(c, f(c)) \in A \bowtie^{f} K$, we have $\left((1,1)-(e, f(e))-\left(c, f(c)((1,1)-(a, f(a)+k)) \in J\left(A \bowtie^{f} K\right)\right.\right.$, by Lemma 2.15 , and the result follows.

Corollary 4.11. Let $K$ be a nil ideal of $B$ and $A$ be an exchange ring. Then $f(A)+K$ is an exchange ring.

By [17, Proposition 2.5], in an exchange ring every central element is the sum of an idempotent and a unit. So if $R$ is an abelian exchange ring, then $C(R)$ is an exchange ring, [17, Corollary 2.6]. By [21], every clean ring is exchange.

A ring with central idempotents (i.e., abelian ring) is clean if and only if it is exchange. Now we investigate necessary and sufficient conditions under which the amalgamated ring $A \bowtie^{f} K$ is abelian.

Proposition 4.12. For the amalgamated ring $A \bowtie^{f} K$, we have the following statements.

(1) If $A$ and $f(A)+K$ are abelian rings, then so is $A \bowtie^{f} K$.

(2) If $A \bowtie^{f} K$ is abelian, then so is $A$.

(3) Let $f: A \rightarrow B$ be a monomorphism. If $f(A)+K$ is abelian, then $A \bowtie^{f} K$ is abelian.

(4) Let $K$ be an ideal with $K \subseteq C(B)$. If $A$ is abelian, then $A \bowtie^{f} K$ is abelian.

Acknowledgment. We are thankful to the referee for the valuable comments and suggestions that have greatly improved the presentation of the paper. 


\section{References}

[1] D.D. Anderson, D. Bennis, B. Fahid and A. Shaiea, On n-trivial extension of rings, Rocky Mountain J. Math. 47, 2439-2511, 2017.

[2] R. Antoine, Examples of Armendariz rings, Comm. Algebra, 38 (11), 4130-4143, 2010.

[3] C. Bakkari and M. Es-Saidi, Nil-clean property in amalgamated algebras along an ideal, Ann. Univ. Ferrara, 65, 15-20, 2019.

[4] M.B. Boisen and P.B. Sheldon, CPI-extension: Over rings of integral domains with special prime spectrum, Canad. J. Math. 29, 722-737, 1977.

[5] G. Călugăreanu, UU rings, Carpathian J. Math. 31 (2), 157-163, 2015.

[6] H. Chen, On strongly J-clean rings, Comm. Algebra, 38 (10), 3790-3804, 2010.

[7] M. Chhiti, N. Mahdou and M. Tamekkante, Clean property in amalgamated algebras along an ideal, Hacet. J. Math. Stat. 44 (1), 41-49, 2015.

[8] Y. Chun, Y.C. Jeon, S. Kang, K.N. Lee and Y. Lee, A concept unifying the Armendariz and NI conditions, Bull. Korean Math. Soc. 48 (1), 115-127, 2011.

[9] P. Crawley and B. Jónsson, Refinements for infinite direct decompositions of algebraic systems, Pacific J. Math. 14, 797-855, 1964.

[10] P. Danchev and T.Y. Lam, Rings with unipotent units, Publ. Math. Debrecen, 88 (3-4), 449-466, 2016.

[11] M. D'Anna, A construction of Gorenstein rings, J. Algebra, 306 (2), 507-519, 2006.

[12] M. D'Anna and M. Fontana, The amalgamated duplication of a ring along a multiplicative-canonical ideal, Ark. Mat. 45 (2), 241-252, 2007.

[13] M. D'Anna and M. Fontana, An amalgamated duplication of a ring along an ideal: the basic properties, J. Algebra Appl. 6 (3), 443-459, 2007.

[14] M. D'Anna, C. A. Finocchiaro and M. Fontana, Amalgamated algebras along an ideal, Commutative algebra and its applications, Walter de Gruyter, Berlin, 241-252, 2009.

[15] A.J. Diesl, Nil clean rings, J. Algebra, 383, 197-211, 2013.

[16] M.F. Dischinger, Sur les anneaux fortement $\pi$-reguliers, C. R. Acad. Sc. Paris, 283, 571-573, 1976.

[17] C.Y. Honga, N. Kimb and Y. Lee, Exchange rings and their extensions, J. Pure Appl. Algebra, 179, 117-126, 2003.

[18] T.Y. Lam, A first course in noncommutative rings, Berlin-Heidelberg-New York: Springer-Verlag, 1991.

[19] M. Nagata, Local Rings, Interscience, New York, 1962.

[20] W.K. Nicholson, Semiregular modules and rings, Canad. J. Math. XXVIII, 1105-1120, 1976.

[21] W.K. Nicholson, Lifting idempotents and exchange rings, Trans. Amer. Math. Soc. 229, 269-278, 1977.

[22] W.K. Nicholson, Strongly clean rings and Fitting's lemma, Comm. Algebra, 27 (8), 3583-3592, 1999.

[23] W.K. Nicholson and Y. Zhou, Rings in which elements are uniquely the sum of an idempotent and a unit. Glasgow Math. J. 46, 227-236, 2004.

[24] S. Sahinkaya, G. Tang and Y. Zhou, Nil-clean group rings, J. Algebra Appl. 16 (5), $1750135,2017$.

[25] J. Stock, On rings whose projective modules have the exchange property, J. Algebra, 103, 437-453, 1986.

[26] A. Tuganbaev, Rings close to regular, Moscow Power Engineering Institute, Technological University, Moscow, Russia 2002.

[27] R.B. Warfield Jr., Exchange rings and decompositions of modules, Math. Ann. 199, 31-36, 1972.

[28] Y. Ye, Semiclean rings, Comm. Algebra, 31 (11), 5609-5625, 2003. 